Rev. salud pública. 14 (1): 41-52, 2012

\title{
Evaluación de la utilidad de un programa de cribado mamográfico en Bogotá, Colombia
}

\section{Evaluating the usefulness of a breast screening program in Bogotá, Colombia}

\author{
Mario A. González-Mariño
}

Universidad Nacional de Colombia. Fundación Universitaria San Martín. Bogotá, Colombia. Universidad de Valladolid. España. marioar90@hotmail.com

Recibido 19 Noviembre 2010/Enviado para Modificación 15 Febrero 2012/Aceptado 23 Febrero 2012

\section{RESUMEN}

Objetivo Evaluar mediante un modelo de simulación la utilidad de un programa organizado de cribado mamográfico en Bogotá, Colombia.

Material y Métodos Diseño análisis de costo-efectividad usando el modelo Markov. Se compara la realización de cribado mamográfico bienal en mujeres de 50 a 69 años con respecto al statu quo en el cual no existe este programa. Se evalúa sobrevida, costos e incrementos en la tasa de costo-efectividad.

Resultados El modelo muestra una reducción de la tasa de mortalidad por cáncer de mama en el esquema de cribado mamográfico de ciclo bienal. Se observa que hay un incremento lineal de los años de vida acumulados desde el tercer ciclo con un ahorro acumulado de costos de tratamiento en las diferentes tasas de cribado generando ahorros al sistema de salud.La Razón de costo por año de vida ganado y Producto interno bruto se hace menor de 3 por encima del $50 \%$ de cobertura (indicando que es una intervención costo-efectiva). El costo medio del programa experimenta una disminución porcentual del 40 \% cuando se llega al ciclo 10. De allí la disminución de este costo es menor.

Conclusiones Según el modelo desarrollado es costo-efectivo adelantar un programa de cribado mamográfico bienal con cobertura mayor al $50 \%$, obteniéndose reducción en la mortalidad que se hace más notoria desde el tercer ciclo del esquema evaluado, obteniendo un ahorro en los recursos queelsistema de salud destina al cáncer de mama.

Palabras Clave: Evaluación de costo-efectividad, cribado, mamografía, mama, neoplasias de la mama (fuente: DeCS, BIREME). 


\section{ABSTRACT}

Objective Using a simulation model for evaluating the usefulness of an organized breast cancer screening program in Bogotá, Colombia

Material and methods The Markov model was used for designing a cost-effectiveness analysis; this was compared to a biennial breast cancer screening for women aged 50 to 69 years old. The disease's natural history, breast screening studies and publications concerning breast cancer were used as input for the model; the number of diagnosed cancer cases, stage distribution and mortality, survival, costs and increased effectiveness rate were also analyzed.

Results The model showed that breast cancer mortality rate decreased in biennial breast cancer screening. Life-years-gained became increased from the third cycle onwards. The ratio between the cost of a life-year-gained and gross domestic product was lower than 3 when more than $50 \%$ of the population were screened (meaning that such program would be cost-effective). The program's lowest average cost was achieved by increasing the number of screening cycles as far as the sixth cycle.

Conclusions The simulation model for studying the impact of changing a breast cancer screening program's coverage for women aged 50 to 69 years led to ascertaining that introducing biennial frequency would be cost-effective when more than $50 \%$ of the population were studied. A lower mortality rate would result, mainly from the third cycle onwards, thereby saving important health system resources in Bogotá, Colombia.

Key Words: Cost effectiveness, analysis, breast, breast neoplasm, mammography, preventative, control (source: $M e S H, N L M)$.

E n Colombia el cáncer de mama es el tumor maligno más frecuente en mujeres luego del cáncer de cuello uterino y es la causa más frecuente de muerte por cáncer en mujeres de 15 a 54 años (1).

Este tipo de cáncer afecta principalmente a mujeres en la menopausia (1), siendo estas mujeres el objetivo principal en los programas de cribado organizado. La prueba recomendada es la mamografía (usualmente asociada con el examen médico) (2-5).

El cribado de cáncer de mama continúa siendo motivo de controversia; existen múltiples estudios sobre el tema, con resultados no siempre coincidentes y con serios cuestionamientos sobre su validez y utilidad(6-9). La controversia existente debe hacer que los países subdesarrollados evalúen la introducción de programas ya que no existe hasta el momento una estrategia de cribado organizado para cáncer de mama que satisfaga todas las exigencias y requerimientos de un programa adecuado a sus propias condiciones el cual va a requerir recursos considerables para ser 
invertidos en estrategias de detección temprana en general, diagnóstico, y tratamiento $(10,11)$.

El riesgo de muerte por cáncer de mama según las regiones muestra una concentración de la mortalidad en los grandes centros urbanos del país, siendo Bogotá la ciudad de Colombia con el mayor número de casos. Esta población es también la primera en incidencia, estimada en el período 1995 a 1999 de 990 casos nuevos, tasa cruda de 31,7 y una tasa ajustada por edad de 41,9 por 100000 mujeres (12).

En Colombia no se recomienda adelantar un cribadoorganizado a nivel Nacional pero se pueden contemplar varios sitios con la infraestructura necesaria para soportar un programa de este tipo, siendo posiblemente Bogotá, la ciudad donde se podría lograr un mayor beneficio dada la mayor incidencia de carcinoma mamario y la existencia de recursos humanos y físicos que garantizan la continuidad en la atención (13).

Dentro de los métodos a considerar para definir la inversión garantizando el mejor resultado con dicho recurso está el análisis de costo-efectividad; en este enfoque económico cuando no se dispone de una situación real se usa con frecuencia el modelo de simulación de Markov el cual mediante la introducción de cifras conocidas o estimadas va a permitir evaluar la posibilidad de una inversión (14).

\section{MATERIAL Y METODOS}

Se desarrolló un modelo Markov con longitud de ciclos de dos años el cual incorpora cuatro procesos interrelacionados: a. La evolución natural del cáncer de mama; b. La detección del cáncer de mama mediante cribado con mamografia; c. El tratamiento y; d. La dinámica de mortalidad por otras causas para simular el impacto del aumento de las coberturas de cribado mamográfico en la mortalidad por cáncer de mama en mujeres de 50 a 69 años en la ciudad de Bogotá, Colombia. Los datos poblacionales corresponden al censo del año 2005 y simula la historia natural del cáncer de mama comparando dos poblacionales hipotéticas, una cuyos integrantes fueron asignadas a mamografía bienal y el otro grupo que identifica la situación actual en la cual no existe un programa de cribado y el acceso a la atención de su patología mamaria se hace por examen médico o hallazgo casual por la paciente. Se modelaron cuatro tipos de estadios de la enfermedad: mujeres 
sanas (sin carcinoma), mujeres con carcinoma temprano, mujeres con carcinoma avanzado y fallecimiento. A su vez, los estadios correspondientes a carcinoma temprano y avanzado se subdividieron así: para el primer caso, en carcinoma temprano sin cribado y con cribado. Este último se caracterizó según el tipo de hallazgo posible en la mamografía, según la clasificación BI-RADS (15) y se consideraron los estados III y IV como cáncer de mama avanzado. El modelo refleja el estado de salud de cada mujer entre los 50 y 69 años. Una mujer puede permanecer en el mismo estado de salud o pasar a otro. La probabilidad de cada resultado determina el número de muertes en cada estado de salud. En el grupo de cribado las mujeres con mamografías anormales van a biopsia o en caso de Bi-rads 3 (hallazgos probablemente benignos) a control mamográfico en 6 meses. Si los cambios en este control permanecen estables, se controla cada 6 meses hasta completar dos años. En la Figura 1, se presentan los escenarios evaluados.

Figura 1. Diagrama de estados

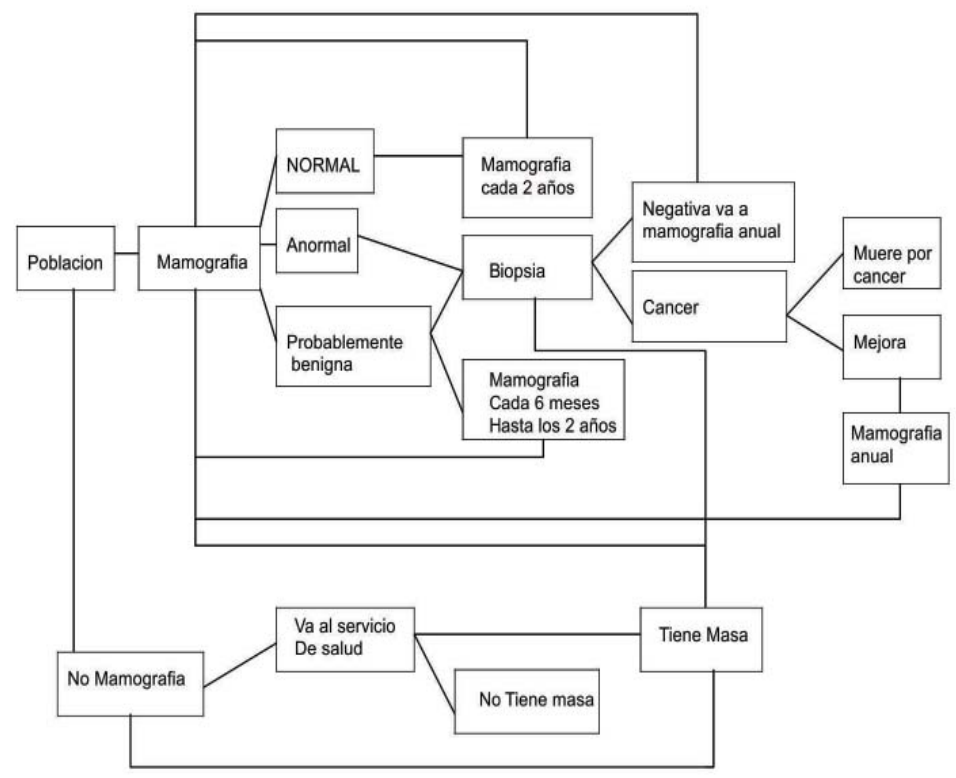

Por basarse esta investigación en un modelo que implica el uso de mamografía, se definirá su ingreso al modelo en los estados tempranos para el grupo de cribado mediante la clasificación del American College of 
Radiology Breast Imaging and Reporting Data System (BI-RADS), mediante el ingreso en tres grupos; los 1 y 2 como normales, los 3 como hallazgos probablemente benignos y los 4 y 5 como anormales.

Se usaron probabilidades de desarrollo de los diferentes eventos de acuerdo con informaciones calculadas o estimadas tanto en el grupo de cribado como de observación. Las pacientes fueron tratadas de acuerdo con los manejos recomendados, asumiendo como resultado final el propuesto para el comportamiento y sobrevida en los diferentes estadios.

En virtud de la ausencia de estudios primarios de buena calidad referidos o realizados en países en vías de desarrollo fue necesario extender la búsqueda a las referencias bibliográficas de los artículos elegidos, privilegiando la inclusión de aquellas de reconocimiento mundial relacionadas con el cribado o el diagnóstico temprano del cáncer de mama. Éstas podían ser de índole observacional, experimental o agregativa (metanálisis, revisiones sistemáticas, guías). Se incluyó información local cuando se encontró presente.

Los costos incluidos fueron los de cribado y los del tratamiento del cáncer. El cálculo de los valores en pesos se obtuvo de información calculada en el año 2004 de los valores de atención promedio en pacientes afectadas por cáncer de mama para el Instituto Nacional de Cancerología.

Se valoro el estudio teniendo en cuenta la perspectiva del sistema de salud. El estudio no considera los costos directos no médicos, como los costos de transporte o desplazamiento, ni tampoco los costos indirectos debido a la pérdida en productividad ocasionada por la enfermedad, tanto del paciente como de los familiares o amigos. Tampoco considera los costos monetarios y no monetarios de los pacientes y sus familiares, para la sociedad y la economía en su conjunto, que suelen ser altos y dolorosos.

Se evaluó la solidez de las conclusiones globales mediante análisis de sensibilidad, cambiando los parámetros individuales y la combinación de parámetros en diferentes rangos.

Para validar los resultados arrojados por el modelo, se simularon escenarios con diferentes coberturas de cribado. 
En el grupo de comparación, statu quo, se tienen en cuenta los datos del registro poblacional de Cali (16), datos del registro institucional de cáncer de la Clínica San Pedro Claver en Bogotá que fueron publicados por el autor de este trabajo $(17,18)$, incidencia estimada de cáncer mamario en Bogotá y otras publicaciones extranjeras

\section{RESULTADOS}

Prevalencia y mortalidad por cáncer de mama según ciclo de cribado Para el primer ciclo se incluyeron 354 cánceres tempranos y 731 cánceres avanzados, estadíos 3 y 4 para un total de 1085 mujeres con cáncer, lo cual conlleva una tasa de prevalencia de 257 cánceres por 100000 mujeres con edades comprendidas entre 50 y 69 años.

Se encuentra que el porcentaje de cánceres avanzados disminuye a medida que se aumentan los ciclos de cobertura pasando porcentualmente del $67,7 \%$ en el primer ciclo al $17,1 \%$ en el décimo ciclo. En el décimo ciclo con una cobertura del $80 \%$, se han ganado 332 años y 1100 años al ciclo 18. En este mismo modelo se incluyeron las probabilidades de detección y el comportamiento del cáncer en mujeres sin cribado mamográfico en las mismas etapas anteriores.

Cambios en cobertura del cribado y mortalidad por cáncer de mama

El aumento de la cobertura del cribado organizado en mujeres de 50 a 69 años para el Distrito Capital muestra una reducción global de la tasa de la mortalidad por cáncer por ciclo bienal que va de 34,61 por $100 \mathrm{mil} / \mathrm{año}$ sin cribado para el primer ciclo "t1" (69,2/2) a 7,8 por 100 mil/año para el ciclo 12 “t12”( $(15,6 / 2)$, con un $80 \%$ de cobertura en el cribado lo que constituye una reducción del 77 $\%$ de la tasa al cabo de 24 años.

Para los dos primeros ciclos, t1 y t2 (4 años), las tasas permanecen invariables a cualquier porcentaje de cobertura. Sin embargo, a partir del tercer ciclo (t3), desde una cobertura del $20 \%$, se inicia una reducción de la mortalidad hasta los niveles ya mencionados.

Se observa en la Figura 2 que la disminución de la mortalidad se consigue de manera importante en los períodos 44 y 7 para la mayoría de coberturas, con poca ganancia adicional sobre las tasas de mortalidad, lo cual incidirá en el 
comportamiento de los costos marginales (definido como el aumento del costo total necesario para producir una unidad adicional del bien, en este caso evitar una muerte). El total de las coberturas han alcanzado aproximadamente entre el 74 y $88 \%$ de las ganancias en disminución de la mortalidad entre el 5 y 6 período (Tabla 1).

Años de vida ganados acumulados

Se encuentra un incremento lineal de los años de vida ganados acumulados desde el tercer ciclo. Así, en el ciclo 12 (año 24), va desde 1250 años para una tasa del $10 \%$, a 8229 años para una tasa de cobertura de cribado del $80 \%$.

Costos del programa

Los costos totales del programa de cribado presentan un incremento lineal relacionado con los aumentos de las tasas de cobertura. Sin embargo, y a pesar de que no se incorporaron ajustes de escala en la producción de servicios, como tampoco cambios en la productividad de los factores, este aumento tiende a ser proporcionalmente menor en la medida que se aumentan los ciclos dentro de cada una de las coberturas (Figura 3).

Figura 2. Mortalidad según cobertura del programa de cribado (Número de muertes por año)

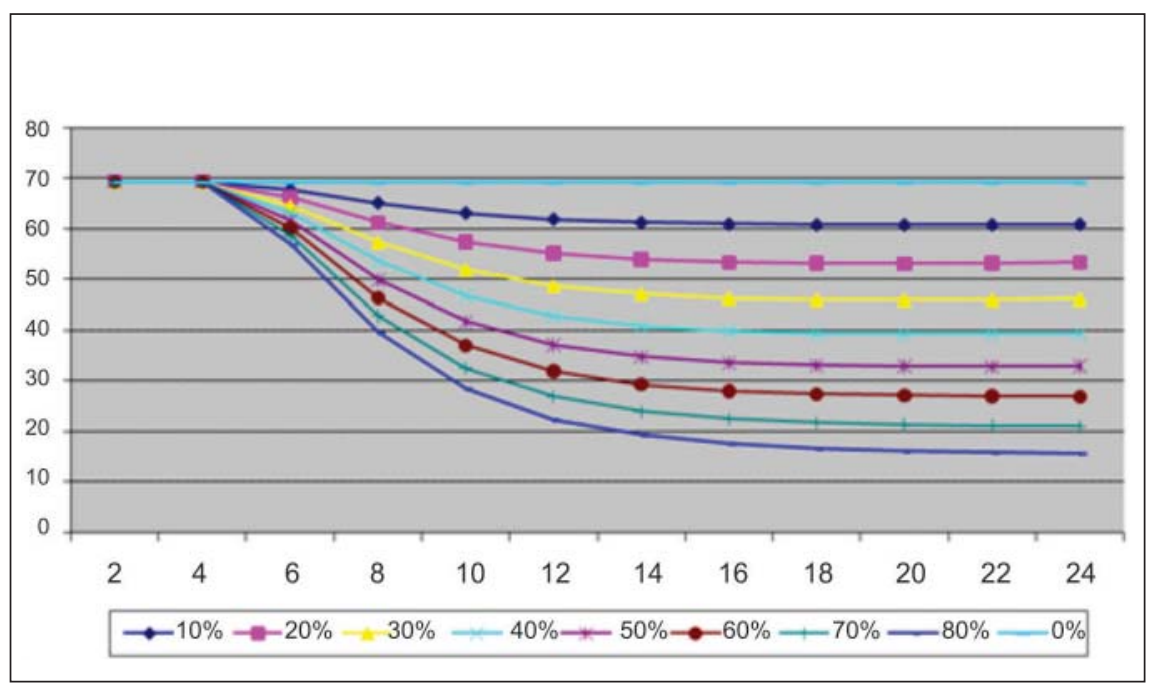


Tabla 1. Frecuencias acumuladas de las ganancias en disminución de la mortalidad por cáncer de mama según diferentes

\begin{tabular}{ccccc}
\multicolumn{4}{c}{ porcentajes de cobertura de la mamografía } \\
\hline \multirow{2}{*}{ Periodo to $t$} & \multicolumn{5}{c}{ Coberturas de la mamografía (\%) } \\
\cline { 2 - 5 } & 20 & 40 & 60 & 80 \\
\hline 1 & 0 & 0 & 0 & 0 \\
2 & 0 & 0 & 0 & 0 \\
3 & 23 & 22 & 22 & 19 \\
4 & 55 & 54 & 54 & 50 \\
5 & 76 & 76 & 76 & 74 \\
6 & 87 & 88 & 88 & 88 \\
7 & 93 & 94 & 94 & 96 \\
8 & 96 & 98 & 98 & 100 \\
9 & 98 & 99 & 99 & 0 \\
10 & 99 & 100 & 100 & 0 \\
11 & 100 & 0 & 0 & 0 \\
12 & 0 & 0 & 0 & 0 \\
\hline
\end{tabular}

Ahorro acumulado de costos de la enfermedad

Se espera que el programa de cribado mejore la identificación de casos tempranos de mayor supervivencia y se requiera menor costo de tratamiento para su logro. En él Se observan ahorros acumulados iniciales de 23 millones de pesos para una cobertura del $10 \%$, en el primer ciclo, hasta 134 mil millones en coberturas del $80 \%$ durante 12 ciclos. Dato que es útil para determinar el costo por año de vida ganado acumulado a diferentes tasas de cobertura de cribado.

Costo por año de vida ganado acumulado

Un año de vida ganado, el primero, tiene un costo similar al costo del programa a una cobertura del $80 \%$ ( $\$ 341541838$ ). Al cabo de 10 ciclos, cada año cuesta 553 veces menos, $\$ 16392160$. Al cabo de 20 ciclos, cuesta aproximadamente 5000 veces menos, \$1 724497 .

Ahorro en costos del programa cribado a una cobertura del $80 \%$

Para el ciclo 12 a una tasa de cobertura del $80 \%$, cada año de vida ganado generaría un ahorro de 2 millones de pesos al sistema de salud, por causa del cáncer de mama. 
Figura 3. Costo promedio del programa vs cobertura (miles de pesos colombianos)

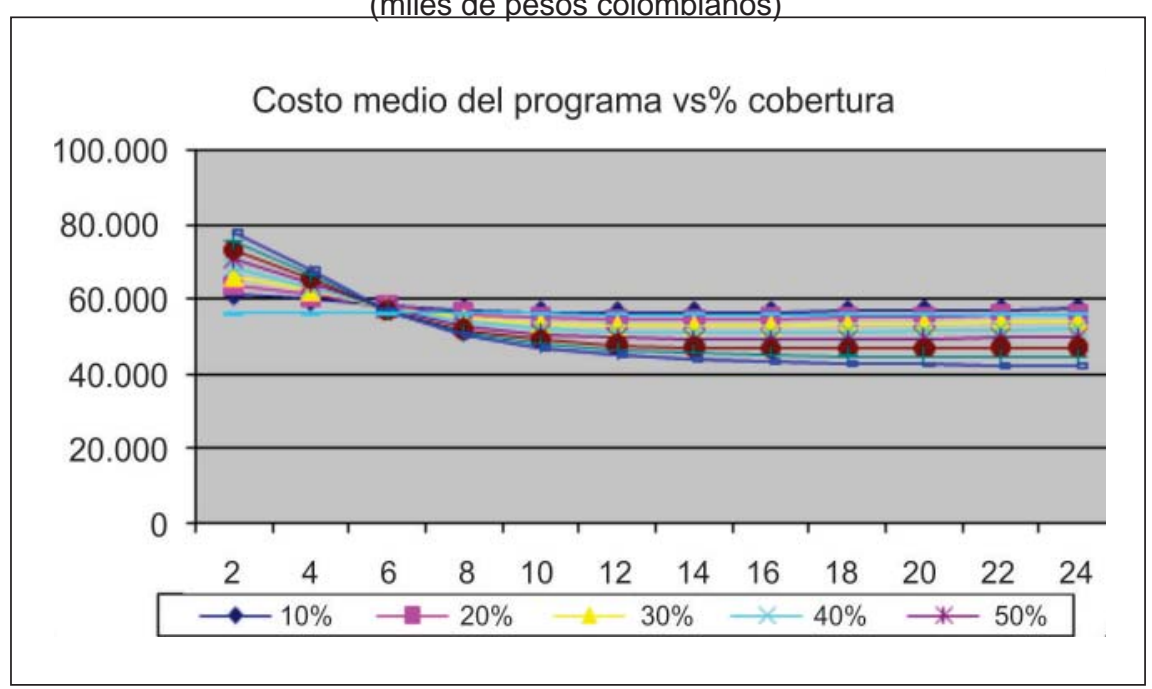

Razón de costo por año de vida ganado y Producto Interno Bruto

Al establecer la relación del costo por año de vida ganado y el producto interno bruto por persona en el 2005 el cual fue de 5232546 en pesos colombianos se puede evidenciar que esta relación se comporta de manera inversamente proporcional con el aumento de la cobertura, por supuesto mostrando que en el origen del dato hay un menor costo por año de vida ganado a medida que se aumenta la cobertura; la Razón de costo por año de vida ganado y Producto interno bruto oscila entre 5,3 y 2,8 al 10 y $80 \%$ de cobertura respectivamente. Esta tendencia se puede evidenciar en la Tabla 2 y la Figura 4. Encontrando que con coberturas por encima del $50 \%$ la relación se hace menor a 3 .

Tabla 2. Razón de costo por año de vida ganado y Producto Interno Bruto por porcentaje de cobertura

\begin{tabular}{ccccccccc}
\hline Cobertura \% & 10 & 20 & 30 & 40 & 50 & 60 & 70 & 80 \\
Razón & 5,3 & 3,8 & 3,3 & 3,1 & 3,0 & 2,9 & 2,9 & 2,8 \\
\hline
\end{tabular}


Figura 4. Razón de costos vs cobertura

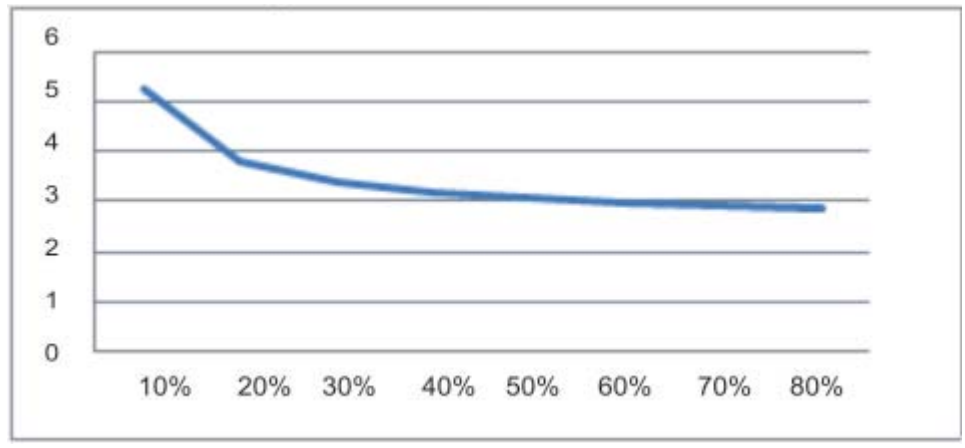

\section{DISCUSIÓN}

Los análisis de costo-efectividad ayudan a orientar decisiones efectivas y eficientes. Esto es, si estamos consiguiendo el máximo retorno humano para los recursos invertidos (19). A pesar de que en Colombia no se tiene un dato estimado de lo que es costo efectivo como si ocurre en los Estados Unidosen donde a los programas de cribado de menos de U.S $\$ 50000$ por año de vida ganado se les considera como favorables (20) se puede usar el parámetro de la Comisión sobre Macroeconomía y Salud, de la Organización Mundial de la Salud (OMS) que se basa en el producto interno bruto (PIB) para establecer umbrales a partir de los cuales puede considerarse una intervención como costo-efectiva (21). La comisión propone las siguientes tres categorías para una intervención: altamente costo-efectiva si la razón de costo por año de vida ganado es menor a una vez el PIB per cápita, costo-efectiva si se encuentra entre una y tres veces el PIB per cápita, y no costo-efectiva si es mayor que tres veces el PIB per cápita. De acuerdo con esto y como se puede apreciar en la Tabla 2 y la Figura 4, el análisis de sensibilidad con una cobertura en el cribado desde el $60 \%$ hace que según este modelo propuesto para estimar el cribado mamográfico en Bogotá, la intervención sea costo-efectiva pero en valores inferiores como en el $50 \%$ ya no lo sea. Estas estimaciones deben tomarse con precaución toda vez que corresponden a escenarios optimistas en cuanto al programa de cribado, en el cual se presupone que las mujeres que ingresan a éste tienen un apego perfecto, es decir, que acuden con la periodicidad indicada y se adhieren perfectamente al seguimiento.

Los costos del programa a medida que se aumenta la cobertura se disminuyen como corresponde a una economía de escala en este caso por 
aumento de infraestructura del programa conjugándose factores como la especialización del trabajo, la mejor utilización del personal administrativo, el uso más eficiente del capital y de los equipos, el poder repartir los costos de publicidad y otros costos de operación del programa. Esta tendencia también se puede evidenciar con el costo marginaldefinido para el programa como el aumento del costo total necesario de una mamografía adicional.

En virtud de la ausencia de evidencia local, se recomienda realizar los esfuerzos necesarios para recopilar información esencial sobre el comportamiento de la enfermedad en el país en los siguientes aspectos: ocurrencia, mortalidad, factores de riesgo, estadio clínico en el momento del diagnóstico, supervivencia, oportunidad diagnóstica y oportunidad terapéutica y se debe fortalecer el sistema de registro, notificación y consolidación de la información, sobre morbilidad, mortalidad, utilización de estudios radiológicos y aspectos económicos de la atención a pacientes con cáncer de mama.

Según el modelo desarrollado para simular el impacto del aumento de las coberturas de cribado mamográfico en la mortalidad por cáncer de mama en mujeres de 50 a 69 años en Bogotá, es costo-efectivo adelantar un programa de cribado mamográfico bienal con cobertura mayor al $50 \%$ de esta población de mujeres, obteniéndose reducción en la mortalidad que se hace más notoria desde el tercer ciclo del esquema evaluado y un ahorro en los recursos queelsistema de salud destina al cáncer de mama.

Se recomienda consolidar el sistema de información para la vigilancia, evaluación y revisión epidemiológica, con el fin de disponer de información confiable y sistemática para la mejoría continua del programa y el aseguramiento de la calidad •

\section{REFERENCIAS}

1.Instituto Nacional de Cancerología. Guías de Práctica Clínica en Enfermedades Neoplásicas; 2001. p. 80-110;156-161.

2. Tabar L, Gad A. Screening for brest cancer: the Swedish trial. Radiology. 1981;138:219-22.

3. Anderson I, Aspegren K, Janzon L, Landberg T, LindholmK, Linell F, et al.Mammographic screening and mortality from breast cancer: the Malmö Mammographic screening trial. BMJ. 1988; 297(6654):943-8.

4. Carney PA, Miglioretti DL, Yankaskas BC, Kerlikowske K, Rosemberg R, Rutter C, et al. Individual and combined effect of age, breast density, and hormone replacement therapy use on the accuracy of screening mammography. Ann Intern med. 2003;138:168-75. 
5. Gilligand FD, Joste N, Stauber PM, Hunt WC, RossenbergRb, Redlich G, et al. Biologic characteristics of interval and screen-detected breast cancers. J Natl Cancer Inst. 2000; 92:743-49.

6. Porter P. Global trends in breast cancer incidence. Salud pública Méx. 2009; 51 (Supp. 2): s141-s146.

7. Gøtzsche PC, Nielsen M. Screening for breast cancer with mammography. Cochrane Database of Systematic Reviews 2009, Issue 4.

8. Gotzche PC, Olsen O. Is screening for breast cancer with mammography justifiable? Lancet. 2000;355:129-33.

9. Frisell J, Lidbrink E, Hellstrom L. Follow after 11 years-update of mortality Results the stockholm mammographic screening trial. Breast Cancer Res Treat. 1997; 45(3):263-70.

10.Shyyan R, Masood S, Badwe RA, Errico KM, Liberman L, Ozmen V, et al. Breast cancer in limited-resource countries: diagnosisand pathology. BreastJ.2006;12(suppl1):S27-37.

11. Dos Santos SI. Prevención del Cáncer. En: Epidemiología del Cáncer: Principios y métodos. Agencia internacional de investigación sobre el cáncer, Lyon, Francia; 1999. pp 377-408.

12. Murillo R, Piñeros M, Hernández G. Atlas de mortalidad por cáncer en Colombia. Bogotá: Instituto Nacional de Cancerología-Instituto Geográfico Agustin Codazzi; 2004.

13. Instituto Nacional de Cancerología, Recomendaciones para la tamización y la detección temprana del cáncer de mama en Colombia. Bogotá: INC; 2006.

14. Rubio-Terrés $C$, Echeverría A. Modelos de Markov: una herramienta útil para el análisis fármaco económico. Pharmacoeconomics. 2006; 3(S2):71-78.

15. American College of Radiology. Breast imaging reporting and data system (Bi-rads). 4th Ed. Reston: American College of Radiology; 2003.

16. Registro poblacional de cáncer de Cali, Facultad de Salud. Universidad del Valle [Internet]. Disponible en:rpcc.univalle.edu.co Consultado en Mayo, 2005.

17. González MA. Cáncer mamario. Registro de cáncer Clínica San Pedro Claver 2003. Rev Colomb Cir. 2005; 20(3):144- 149.

18. González MA. Cáncer de seno en la Clínica San Pedro Claver, 2004. Revista de salud pública (Bogotá) 2006; 8(2):163- 169

19. Berry DA, Cronin KA, Plevritis SK, Fryback DG, Clarke L, Zelen M, Mandelblatt JS, Yakovlev AY, Habbema JD, Feuer EJ. Effect of screening and adjuvant therapy on mortality from breast cancer. N Engl J Med. 2005, 353:1784-1792.

20. Kerlikowske K, Salzman P, Phillips KA, Cauley JA, Cummings SR. Continuing screening mammography in women aged 70- 79 years impact on life expectancy and costeffectiveness JAMA. 1999; 282 (22): 2 156-63.

21. Organización Mundial de la Salud (OMS) [Internet]. Eligiendo intervenciones que son costo efectivas, umbrales de costo efectividad. Disponible en: http://www.who.int/choice/ costs/CER_thresholds/en/index.html Consultado: octubre 2008. 